\title{
Diabetes and Bone
}

\author{
Serge Ferrari ${ }^{1}$
}

Received: 22 December 2016 / Accepted: 4 January 2017 / Published online: 8 February 2017

(C) Springer Science+Business Media New York 2017

Diabetes and osteoporosis are two of the most common chronic disorders which prevalence increases worldwide, eventually affecting hundreds of millions of people. The environmental, primarily nutritional, conditions that predispose to diabetes and osteoporosis, respectively, appear quite different, but there may be some common genetic factors predisposing to both disorders. Although the morphotype of subjects developing type 2 diabetes as a consequence of overweight and the metabolic syndrome seems protective against fractures, and is certainly far from the image of the frail elderly with bone fragility, type 2 diabetes is increasingly recognized as an independent risk factor for fractures - at least in the bone community, although not yet broadly recognized as a complication of glucose impairment in the diabetes community. The risk of fragility fractures is even higher among the leaner patients with type 1 diabetes, whose long-standing disease is associated with an up to fivefold higher hip fracture risk, which incidence starts to rise 10-15 years before the exponential rise observed in the non-diabetic population. The relative contribution of decreased bone strength, in particular bone "quality," and increased incidence of injurious falls, to the higher fracture risk among diabetics remains unknown.

The first descriptions of decreased skeletal mass and impaired bone development in children with diabetes, and of vertebral crush fractures in diabetic patients from the Joslin Clinic, date back almost a century. Yet the full mechanisms for such bone fragility are so complex that they

Serge Ferrari

serge.ferrari@unige.ch

1 Service and Laboratory of Bone Diseases, Geneva University Hospital (HUG) and Faculty of Medicine, 1205 Geneva, Switzerland only begin to be understood and have not yet been fully integrated $[1,2]$. In particular, the relative contribution of altered bone microstructure and material properties versus bone mass in diabetes-related fractures has not yet been unequivocably proven. An intriguing question is whether there is really increased cortical porosity in diabetes, and if so, what are the mechanisms involved in consideration of the fact that poor glucose control has been associated with lower, rather than higher, bone turnover. Even more intriguing are recent reports suggesting that the bone alterations in diabetes are more prominent among subjects with microvascular complications, suggesting that impairment of vascularization to the skeleton, in particular osteoblasts and osteocytes, could play an important role in the pathophysiology of bone fragility in diabetes. The relative paucity of bone biopsy studies in this area certainly does not help clarify whether bone fragility in diabetes is the expression of alterations that mirror those found in common osteoporosis, albeit perhaps in different proportions (bone quality changes disproportionately greater than bone mineral mass changes) - in which case we could qualify this bone fragility as "diabetoporosis (DIO)," by analogy to "GIOP," or whether specific alterations in the bone/bone marrow of diabetic patients occur that are not found in common osteoporosis, in which case we should call this type of bone fragility "diabetic bone disease (DBD)," by analogy to CKD-MBD. Another key question is regarding the role of osteocytes in this disorder, as they are involved in both the control of bone modeling and remodeling and in glucose homeostasis.

From a clinical standpoint, there are many challenges as well. Both aBMD and FRAX underestimate fracture prediction in diabetic patients, particularly with type 2, and the addition of TBS brings only a marginal improvement to this evaluation. Hence, new clinical tools and adjusted 
algorithms will be necessary. This task will be even more complicated to achieve that the numerous treatments of diabetes have differential effects on bone homeostasis and fracture risk and will therefore have to be integrated as well. But an even more challenging task concerns the pharmacological treatment of bone fragility in diabetes. Indeed, there is currently no study that has evaluated any of the osteoporosis drugs effects on bone strength and fracture outcomes in this context. The few available data are about women entering osteoporosis trials and belonging to a subgroup of subjects with diabetes at baseline. However, we completely ignore whether these drugs will be equally effective, and safe, in the absence of low bone mass but prominent alterations of bone "quality." Dedicated trials are therefore needed, as are more studies to eventually identify new/specific bone markers, capable to improve the evaluation of fracture risk and treatment response in these subjects.

This special issue of CTI covers in many great details the complexities, challenges, and opportunities of bone fragility in diabetes. Abrahamsen et al. present the epidemiology of fractures associated with diabetes, while Leslie and coll. discuss the difficulties to evaluate fracture risk in diabetes using the usual prediction tools such as BMD and FRAX.
Napoli et al. summarize the multiple biological pathways involved in the pathophysiology of bone fragility associated with diabetes, while Bonnet looks into the reverse, and somehow controversial, relationship, namely how bone controls glucose homeostasis. Eventually Schwartz et al. and Chandran review, respectively, the effects of osteoporosis drugs in diabetes and the effects of diabetes drugs on bone.

As such this issue should be both an eye-opener for all of those taking care of these patients and a trigger to pursue and develop the many experimental and clinical studies that are needed to improve the understanding and management of this increasingly common condition.

\section{References}

1. Hough FS, Pierroz D, Cooper C, Ferrari S, The IOF CSA Bone and Diabetes Working Group (2016) Mechanisms and evaluation of bone fragility in type 1 diabetes mellitus. Eur $\mathrm{J}$ Endocrinol 174(4):R127-R138

2. Nicola N, Chandran M, Pierroz DD, Abrahamsen B, Schwartz AV, Ferrari SL, IOF Bone and Diabetes Working Group (2016) Mechanisms of diabetes-induced bone fragility. Nat Rev Endocrinol. doi:10.1038/nrendo.2016.153 